\title{
¿Es necesario un programa nacional de identificación de la morbilidad materna grave en Chile?
}

Reducir la mortalidad materna (MM) se mantiene como una prioridad global para el desarrollo sustentable. Los Objetivos de Desarrollo del Milenio incluyeron la reducción de la razón de mortalidad materna a tres cuartos de la observada en 1990 para el año 2015 (1). Se estima que la tendencia de la reducción global de la razón de MM promedia el 1,3\% anual en el periodo 1990-2013, situación que está lejos de la meta de 5,5\% anual necesaria para lograr el objetivo propuesto (2). En el año 2015 las Naciones Unidas fijó los nuevos "Objetivos para el Desarrollo Sustentable" para el 2030 que incluyen eliminar la MM prevenible con una meta de reducción de $2 / 3$ la existente en el año 2010 dando énfasis en lograr equidad en poblaciones vulnerables. Lo anterior implica, para naciones como Chile, lograr una reducción promedio anual del $5,5 \%$ durante el periodo para poder cumplir la meta (3).

Chile presenta una tendencia secular hacia reducción de la MM $(4,5)$. Un descenso de la razón de MM de $60 \%$ durante el periodo $1990-2013$ es muy positivo a nivel regional (6). Sin embargo, esto refleja primariamente la reducción observada en el primer decenio sin registrarse cambios significativos en los últimos 10 años. Aun considerando la reducción observada desde 1990 , no se espera que Chile alcance la meta del 2015 en los próximos 15 años (2), a menos que se implementen nuevas estrategias.

Entre las iniciativas dirigidas para acelerar la reducción se encuentra el monitoreo de Morbilidad Materna Grave (MMG) como indicador y el análisis de los factores asociados a MMG como sistema de mejoramiento continuo aplicable a un centro o a un sistema de salud.

Desde el año 2008 la OMS ha impulsado un proceso sistemático para el desarrollo de la definición y clasificación de MMG. Bajo este modelo, se entiende que la morbilidad materna comprende un espectro de evoluciones de las embarazadas y puérperas, donde su resultado depende de la presencia de complicación y de la magnitud de la amenaza vital. De esta manera, la característica común entre los casos de sobrevivientes y los casos de muerte sería una complicación con amenaza vital. Así un caso de MMG, sobreviviente es definida como "Una mujer que casi muere pero sobrevive a una complicación que ocurre durante el embarazo, el parto o dentro de los 42 días de terminado el embarazo"(7).

Los criterios OMS, capaces de identificar en forma confiable a los casos de MMG, han cumplido varias fases: 1) Un proceso de desarrollo de los criterios informado por evidencia. 2) Un proceso de validación en escenarios restringidos y en escenarios amplios donde los criterios OMS de MMG presentan una óptima concordancia mayor al azar con otro sistema diferente para la detección de insuficiencia de órganos. A su vez, los estudios de validación muestran una alta sensibilidad y especificidad para la detección de los casos de muerte materna. En escenarios amplios de maternidades en Brasil se estimó el cociente de verosimilitud test positivo de 106,8 IC95\% $(99,6$ a 114,6$)$ y cociente de verosimilitud test negativo de 0,0 para muerte materna. La evidencia sobre el desarrollo y rendimiento de los criterios basados en insuficiencia de órganos para definir MMG desarrollados por OMS es consistente y han sido promovidos para su aplicación a nivel regional por la Organización Panamericana de la Salud y el Centro Latinoamericano de Estudios Perinatales.

A nivel global las estimaciones de prevalencia de MMG dependen de los criterios utilizados para clasificar los casos. Se han utilizado criterios basados en síntomas y signos de enfermedad, criterios basados en el manejo y criterios basados en insuficiencia de órganos para clasificar los casos. Los criterios con mayor confiabilidad y aplicabilidad corresponden a los criterios asociados a insuficiencia de órganos y de acuerdo a estos se estima que la prevalencia global de MMG es de 4,2 por 1.000 partos (8). Además se estima que la razón de MMG por cada muerte materna de 4:1 a 6:1 bajo criterios de disfunción de órganos (9).

En Chile la información sobre prevalencia de MMG es indirecta y está limitada a series de casos 
en centros hospitalarios. Dos estudios retrospectivos describen la prevalencia de histerectomía de emergencia estimada en 1 a 2,2 por 1.000 partos $(10,11)$ y un estudio describe la prevalencia de ingreso a $\mathrm{UCl}$ en un hospital universitario estimada en 11,8 por 1.000 partos (12). Lo anterior resalta la necesidad de estudios que evalúen la prevalencia y distribución de causas de MMG con metodología estandarizada a nivel nacional.

La evidencia sobre la utilidad de implementación de sistemas de reporte para auditoría y retroalimentación con el objetivo de mejorar la morbilidad o mortalidad materna o perinatal es escasa, sin estudios de asignación aleatoria existentes, la evaluación de su utilidad está basada en estudios observacionales y opinión de expertos. Sistemas de notificación confidencial nacionales se mantienen en países que tienen muy baja MM, sistemas de reporte y auditoría para MMG han sido implementados recientemente en varios países de diversa tasa de MM con el objetivo de acelerar su reducción como en Escocia y Colombia. La OMS, OPS, el CLAP, la FLASOG, ACOG, junto con otras entidades promueven la implementación de un sistema de aprendizaje basado en el reporte voluntario de MMG que asegure la confidencialidad de la información alineado con la normativa de calidad y seguridad asistencial a nivel de centro o sistema de salud.

Para la detección de los casos de MMG se requiere de una primera etapa de identificación sistemática de los casos de potencial amenaza vital basado en criterios clínicos. Diversas estrategias han sido descritas para la implementación de esta fase involucrando a agentes claves, personal dedicado con visitas diarias a entregas de turno, salas de parto, unidades de emergencia, etc. Una vez detectados los casos potenciales de MMG deben ser confirmados según los criterios de insuficiencia de órganos considerando la información clínica y la proveída por lo involucrados en caso necesario.

La información recolectada en torno a un caso debe contribuir a un ciclo de mejora continua basada en el aprendizaje de la auditoría clínica de los casos de MMG. Con una mirada sistémica, que permita la identificación de oportunidades y obstáculos para la mejoría del cuidado, implementación de intervenciones en múltiples niveles adaptadas a la realidad local y monitoreo de su impacto a través de la vigilancia de indicadores.

Basados en la propuesta de la OMS y experiencias publicadas, 2 niveles de análisis son recomendables:

- A nivel local el establecimiento de un Comité Institucional de Análisis Confidencial de MMG interdisciplinario, que tenga miembros expertos de los estamentos involucrados en la atención perinatal y del programa de Calidad y Seguridad institucional, cuyo rol corresponde clasificar el caso de MMG, completar el formulario de registro de MMG, analizar la información obtenida bajo los lineamientos de sistemas de aprendizaje con énfasis en las mejoras de sistema, consistentes con análisis causa-raíz o protocolo de Londres (13), del cual se genera una conclusión sobre las oportunidades de mejoría y recomendaciones específicas que cada institución acuerda como implementar. Así también, tendría a cargo la elaboración de informes periódicos locales y reportes a nivel nacional.

- A nivel nacional un sistema de reporte voluntario institucional de casos de MMG no identificados, que permita mantener un monitoreo de su prevalencia y del uso de intervenciones claves, consistente con el modelo propuesto por OMS y externalizar las oportunidades de mejora e intervenciones efectivas tanto a nivel nacional como internacional.

El sistema de reporte y mejoría continua que sea implementado tanto a nivel local como nacional requiere de normativas y regulaciones legales específicas que resguarden la confidencialidad de la información sin identificación local de los casos y favorezcan un ámbito de reporte seguro en un marco general regulado, tal como ha sido recomendado por diversas entidades internacionales como supranacionales (14). En ese contexto la implementación de un programa nacional de identificación de la morbilidad materna grave podría ser un aporte tanto a la reducción de la razón de mortalidad materna como de la morbilidad materna grave, junto con proveer de un marco para una cultura de mejoría continua de la salud materna y perinatal.

Dr. Claudio Vera PG. Jefe Unidad de Medicina Materno-Fetal. División de Obstetricia y Ginecología. Escuela de Medicina. Universidad Católica de Chile.

\section{REFERENCIAS}

1. United Nations. United Nations Millennium Declaration. Resolution adopted by the General Assembly, 55th Session of the United Nations General Assembly2000 18 de Septiembre de 2015.

2. Kassebaum NJ, Bertozzi-Villa A, Coggeshall MS, Shackelford KA, Steiner C, Heuton KR, et al. Global, regional, and national levels and causes of maternal mortality during 1990-2013: a systematic analysis for the Global Burden of Disease Study 2013. Lancet. 2014;384(9947):980-1004.

3. World Health Organization. Strategies toward ending preventable maternal mortality (EPMM) 2015. Disponible en: http://www.who.int/reproductivehealth/topics/maternal_perinatal/epmm/en/.

4. Donoso Siña $\mathrm{E}$. [The reduction in maternal mortality in Chile, 1990-2000]. Rev Panam Salud Publica. 2004;15(5):326-30.

5. Donoso E, Carvajal JA. [The change in the epidemiological profile of maternal mortality in Chile will hinder 
the fulfillment of the Millennium 5th goal]. Rev Med Chil. 2012;140(10):1253-62.

6. World Health Organization. Trends in maternal mortality: 1990 to 2013 by WHO, UNICEF, UNFPA, The World Bank and the United Nations Population Division. 2014.

7. World Health Organization. Evaluating the quality of care for severe pregnancy complications: the WHO near-miss approach for maternal health. 2011.

8. Tunçalp O, Hindin MJ, Souza JP, Chou D, Say L. The prevalence of maternal near miss: a systematic review. BJOG 2012;119(6):653-61.

9. Souza JP, Cecatti JG, Parpinelli MA, de Sousa $\mathrm{MH}$, Serruya SJ. [Systematic review of near miss maternal morbidity]. Cadernos de saude publica. 2006;22(2):255-64.

10. Robert JA RP, Rodriguez T, Pons A. Histerectomía postparto: Experiencia de Clínica Las Condes. Revista chilena de Obstetricia y Ginecologia. 2010;75(5):3005.
11. Aguilera B SK, Lattus J, Pantoja V, Moscoso F, Gulchard C, et al. Histerectomía obstétrica de emergencia. Experiencia de el Hospital Santiago Oriente Dr. Luís Tisné Brousse. Rev Obstet Ginecol - Hosp Santiago Oriente Dr Luís Tisné Brousse. 2006;1(1):39-44.

12. Hasbún J, Sepúlveda-Martínez A, Cornejo R, Romero C. Morbilidad materna grave e ingreso a cuidado intensivo: Hospital Clínico Universidad de Chile (20062010). Rev Med Chile 2010;141(12):1512-9.

13. Taylor- Adams S, Vincent C. Systems analysis of clinicals incidents. The London protocols. Disponible en: https://www1.imperial.ac.uk/resources/ C85B6574-7E28-4BE6-BE61-E94C3F6243CE/ londonprotocol_e.pdf.

14. Organización Panamericana de la Salud. Análisis comparado del marco jurídico sobre Calidad de la Atención y Seguridad del Paciente (CASP) en América Latina, España y la Comunidad Europea. Washington, DC: OPS; 2012. 